\title{
Distributed Fault Diagnosis using Sensor Networks and Consensus-based Filters
}

\author{
Elisa Franco, Reza Olfati-Saber, Thomas Parisini, and Marios M. Polycarpou
}

\begin{abstract}
This paper considers the problem of designing distributed fault diagnosis algorithms for dynamic systems using sensor networks. A network of distributed estimation agents is designed where a bank of local Kalman filters is embedded into each sensor. The diagnosis decision is performed by a distributed hypothesis testing method that relies on a belief consensus algorithm. Under certain assumptions, both the distributed estimation and the diagnosis algorithms are derived from their centralized counterparts thanks to dynamic average-consensus techniques. Simulation results are provided to demonstrate the effectiveness of the proposed architecture and algorithm.
\end{abstract}

\section{INTRODUCTION}

Modern industrial systems often operate in challenging conditions, increasing the possibility of system faults characterized by critical and unpredictable changes in the system dynamics. Moreover, several systems of practical interest are large-scale and/or physically distributed: sensor networks made of low-cost monitoring sensors represent an effective solution for distributed fault diagnosis.

Over the last two decades, the design and analysis of fault-diagnosis algorithms using the model-based analytical redundancy approach have received significant attention [3], [6], [7]. However, most of the available methods make use of state-estimation techniques where the information obtained from the sensing devices are centralized (see, foor instance, [17]). In this respect, apart from a few works, links between fault diagnosis and distributed estimation methodologies are lacking. A notable exception is [5], where the authors propose a decentralized implementation of the Fault Detection Filters technique on a Linear Time Invariant (LTI) system, to be monitored through a network of sensors. Such filters are a special class of estimators: assuming an all-to-all interconnection topology (each sensor is directly connected to all the other sensors), previous results on decentralized Kalman filtering [4] can be exploited in order to perform decentralized Fault Detection and Isolation (FDI). The assumption of a fully-connected network topology though leads to a communication complexity cost of $\mathcal{O}\left(n^{2}\right)$ that in practice is not scalable when $n$ is large, where $n$ is the number of

Corresponding author: Thomas Parisini. This work has been partially supported by the Italian Ministry for University and Research.

E. Franco is with the Dept. of Control and Dynamical Systems, California Institute of Technology, Pasadena CA. (\{elisa\}@cds.caltech.edu)

Reza Olfati-Saber is with the Thayer School of Engineering, Dartmouth College, Hanover, NH 03755, USA (olfati@dartmouth.edu)

T. Parisini is with the Dept. of Electrical, Electronic and Computer Engineering, DEEI-University of Trieste, Italy. (\{parisini\}@units.it)

M. M. Polycarpou is with the Dept. of Electrical and Computer Engineering, University of Cyprus, Nicosia 1678, Cyprus. (mpolycar@ucy.ac.cy) nodes. Furthermore, new approaches using particle filters in the field of distributed estimation applied to hybrid systems have been proposed in [11], [6]; another important work focusing on complex systems is [10].

A novel and completely different distributed estimation technique has been introduced very recently in [9] where a centralized Kalman Filter (KF) is shown to be equivalent to a set of micro Kalman filters (MKF)) each embedded in one node of a sensor network (in [9]. This equivalence is established using consensus algorithms [11], [12] that only require a sparse but connected network, reducing the communication complexity to $\mathcal{O}(n \log n)$ (or $\mathcal{O}(n)$ for planar graphs).

In this paper, a distributed fault detection and isolation (FDI) methodology is described, relying on the aforementioned decentralized Kalman state-estimation technique. In the literature, several works can be found dealing with fault diagnosis using Kalman filters (see, for instance, [8], [1],[15]). The innovation sequences of a KF reflect model mismatches generated by the occurrence of a fault at the level of the system parameters. We propose a detection procedure based on the analysis of the innovations of a KF using the nominal model of the system to be monitored; once a fault is detected, a multiple model estimation based isolation scheme takes place. The innovations generated by a bank of KF's, each endowed with a different faulty model of the target system, are processed with a suitable hypothesis testing algorithm. Unlike previous works in a centralized setting, also the hypothesis testing methodology is distributed over the sensor network. Bayesian networks and belief propagation, introduced in [13], have proven to be an effective analytical tool for distributed information processing and fusion. In [14], a decentralized hypothesis testing approach is provided for fully-connected networks. This drawback in communication cost has recently been overcome in [10] by formulating the problem of multi-hypothesis testing as an average-consensus problem that can be efficiently solved using a scalable algorithm. Such method is applicable to this setting, by assuming the innovation sequences generated by the MKF's to be sufficiently uncorrelated. The most likely model is chosen as the one with the maximum conditional probability.

In the next section, the distributed fault diagnosis problem is stated, whereas in Section III, the distributed estimation methodology and the data fusion problem are presented. Section IV addresses the distributed detection and isolation algorithm and Section $\mathrm{V}$ reports some early simulation results. 


\section{Distributed FAUlT DETECTION PROBLEM}

Consider the following model of the plant to be monitored:

$$
x(t+1)=A x(t)+G \xi(t)+\beta(t-\bar{t}) \Phi x(t)
$$

where $t \geq 0, x \in \mathbb{R}^{n}$ denotes the state vector and $\xi \sim W G N(0, \Xi)$ denotes a Gaussian zero-mean white noise describing the disturbances affecting the state vector. According to the FDI framework proposed in [16], the term $\beta(t-\bar{t}) \Phi x(t)$ characterizes an additive fault occurring at time $\bar{t}$ and affecting the structure of system's dynamics. In general, $\beta(t-\bar{t})$ is a scalar function representing the time profiles of the faults occurring at some unknown time $\bar{t}$. In this paper, we consider abrupt faults modeled by: $\beta(t-\bar{t})=0$ if $t<\bar{t}$ and $\beta(t-\bar{t})=1$ if $t \geq \bar{t}$. For isolation purposes, we assume that there are $N_{f}$ possible fault matrices: $\Phi$ belongs to a finite set of known matrices given by $\mathcal{F} \triangleq\left\{\Phi^{1}, \ldots, \Phi^{N_{f}}\right\}$. Therefore the system evolves with model:

$$
x(t+1)=F x(t)+G \xi(t),
$$

where, at each time-instant $t, F$ takes on the form of one matrix in the set $\left\{A, A+\Phi^{1}, \ldots, A+\Phi^{N_{f}}\right\}$. Obviously $F=A$ for $t<\bar{t}$. Throughout the paper, we make the following assumption:

Assumption 1: Only one fault may occur at a given time.

A sensor network of $N$ estimation agents $\mathcal{E} \triangleq$ $\left\{\mathcal{E}^{i}: i=1, \ldots, N\right\}$ will be specifically designed to perform a distributed FDI task. In particular, each $\mathcal{E}^{i}$ will use a local measurement $y^{i}$ of the accessible output of the plant, denoted by $y$ (Fig. 1).

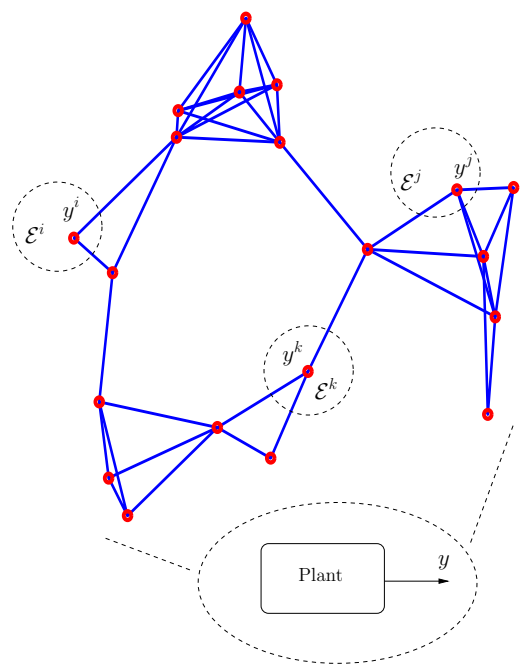

Fig. 1. FD sensor network framework.

Each $\mathcal{E}^{i}$ is networked with a subset of other agents or neighbors, that will be identified by a set of indexes $\mathcal{M}^{i} \subset$ $\{1, \ldots, N\} \backslash\{i\}$. The network of diagnostic estimation agents can be topologically described as a graph $\mathcal{G}=(\mathcal{V}, \mathcal{E})$, i.e. a set of $N$ vertices $\mathcal{V}$ and edges $\mathcal{E}$ representing the sensors and the communication flow between them, respectively. Some useful quantities associated with the graph $\mathcal{G}$ are the adjacency matrix, $\mathcal{A}$, whose elements $a^{i j}$ are nonzero only whenever $(i, j) \in E$; the degree matrix $\Delta=\Delta(\mathcal{A})$, defined as a diagonal matrix whose elements are $d^{i i}=\sum_{j \in \mathcal{M}^{i}} a^{i j}$; finally the Laplacian associated with the network topology, defined as $\mathcal{L} \triangleq \Delta-\mathcal{A}$. The properties of the Laplacian of a graph [2] are very important in terms of agreement (or synchronization) of all its elements: such task is required for the network of estimators, as will be pointed out in the next section.

Assumption 2: The estimation agents network is described by an overlay graph which is undirected, i.e. the adjacency matrix is symmetric $\mathcal{A}=\mathcal{A}^{\top}$, and connected: given any two nodes $i, j$ of the graph, with $i \neq j$, there always exists a path connecting such nodes.

Several advantages are given by the use of a network of monitoring units over a single one: the proposed approach will be demonstrated to efficiently break down in simpler computational units the burden of estimation and detection, also minimizing the communication requirements. The network of estimation agents performs essentially three tasks:

1) Distributed multiple model estimation. A bank of $N_{f}+1$ micro-Kalman filters is designed into each monitoring agent for FDI purposes.

2) Data fusion. Each estimation agent $\mathcal{E}^{i}, i=1, \ldots, N$ is endowed with data fusion algorithms which will be necessary in order to distribute the estimation process.

3) Fault detection and isolation. The system state $x(t), t \geq 0$ is estimated by using the nominal model under healthy mode of behavior; when a fault is detected, the remaining $N_{f}$ filters are activated for the purpose of fault isolation. When the isolation filters are activated, a distributed belief propagation algorithm will be implemented, in order to synchronize the network on the final diagnostic decision.

Tasks 1), 2), and 3) will make extensive use of consensus or agreement algorithms (see [11] and the references cited therein). These techniques will be discussed in the next section where the distributed estimation methodology over the sensor network will be described.

\section{SENSOR NETWORK DISTRIBUTED ESTIMATION}

Let us recall from the previous section, that each estimation agent $\mathcal{E}^{i}, i=1, \ldots, N$ performs the tasks of distributed estimation, data fusion, and belief propagation/FDI. In this section, the first two tasks will be described in some detail, whereas, in Section IV, the third task will be addressed.

\section{A. Distributed multiple model estimation}

We assume that each estimation agent $\mathcal{E}^{i}$ has $N_{f}+1$ models of the system:

$$
\begin{aligned}
x(t+1) & =F x(t)+G \xi(t) \\
y^{i}(t) & =H^{i} x(t)+v^{i}(t),
\end{aligned}
$$


where $F \in\left\{A, A+\Phi^{1}, \ldots, A+\Phi^{N_{f}}\right\} ; y^{i} \in \mathbb{R}^{p}$ is the output of system (2) measured by the $i$ th sensor, matrix $H^{i}$ is known to the estimation agent $\mathcal{E}^{i}$, and $v^{i} \sim W G N\left(0, V^{i}\right)$. The following assumption is also in place:

Assumption 3: The measurement noise $v^{i}(t)$ of estimation agent $\mathcal{E}^{i}$ is not correlated with the measurement noise of estimation agent $\mathcal{E}^{j}, v^{j}(t), \forall i, j, i \neq j$.

The interested reader can from this point follow step by step [9] to derive the equations of the distributed KF. We do not report such procedure due to space limitations, but only recall that the two key quantities to distribute the KF computation are the average covariance matrix:

$$
S \triangleq \frac{1}{N} \sum_{i=1}^{N} H^{i \top} V^{i-1} H^{i}
$$

and the average measurement vector

$$
y(t) \triangleq \frac{1}{N} \sum_{i=1}^{N} H^{i \top} V^{i-1} y^{i}(t) .
$$

By construction (4) and (5) do not depend on the specific $i$ th estimation agent and can therefore be computed reaching an agreement (or consensus). This specific aspect of the estimation process falls into the data fusion task and will be discussed in Subsection III-B. For now, assume that matrix $S$ and vector $y(t)$ can be computed in a distributed way. Under such assumption, for each different model of the system, a specific MKF can be implemented on the $i$ th estimation agent, as proved in [9]. The equations of such filter are:

$$
\begin{aligned}
& M_{\mu}(t)=\left[P_{\mu}^{-1}(t)+S\right]^{-1}, \\
& \hat{x}(t)=\bar{x}(t)+M_{\mu}(t)[y(t)-S \bar{x}(t)], \\
& P_{\mu}(t+1)=F M_{\mu}(t) F^{\top}+G \Xi G^{\top}, \\
& \bar{x}(t+1)=F \hat{x}(t),
\end{aligned}
$$

where we denote by $\bar{x}(t)$ and by $\hat{x}(t)$ the a priori and a posteriori state estimates; we set $P_{\mu}(0) \triangleq M_{\mu}(0) P(0)$, where $P(0)$ is the initial state estimation error covariance, and $M_{\mu}(0)=P_{\mu}(0)$. The above update equations for the error covariance and for the state estimation are common to each agent for a specific filter using model $F=A+\Phi^{j}$. Note that despite using different models, all the filters need the same averaged quantities $y(t)$ and $S$.

We introduced the KF-based multiple model estimation procedure: as we will detail in Section IV, each agent estimates at all times the system state using the nominal model $F=A$. This filter will be used as a detection filter. When a failure is detected, the other filters will be turned on, and used as isolation filters to reach a decision on the type of fault occurred.

\section{B. Data fusion}

It is important to note that the case of all-to-all connectivity of the network trivially requires one step to compute an average of $N$ known quantities. Far more interesting is when such quantities are time varying and the graph is simply connected. In our specific problem, (4) and (5) will be calculated using the consensus filters introduced in [12]: (4) needs to be computed using a Band Pass Consensus Filter (BCF), while to compute (5) we need a Low Pass Consensus Filter (LCF). Consensus filters are dynamic distributed averaging algorithims that are capable of averaging time-varying signals. The discretization step-size $T$ of the consensus filters and the plant (1) are equal. Unlike static average-consensus algorithms, there is no need to wait for the algorithm to approximately converge within a period of $T$, i.e. consensus filters are applicable to relatively faster systems (see [12] for a description of the BCF and LCF filters in a continuoustime setting).

To get some more insight on this issue, let us consider the distributed computation of $y(t)$ defined in (5): the discrete time version of the LCF proposed in [12] will be derived. The generic agent $\mathcal{E}^{i}$ measures $y^{i}(t)$ and can easily compute $H^{i \top} V^{i-1} y^{i}(t)$ while the objective of the network is to track $y(t)$ at each node. For every $\mathcal{E}^{i}, i=1, \ldots, N$, we introduce for the LCF an internal state $z^{i}(t)$, whose update equation is: $z^{i}(t+1)=z^{i}(t)+\epsilon \sum_{j \in \mathcal{M}^{i}}\left[z^{j}(t)-z^{i}(t)\right]+$ $\epsilon \sum_{j \in\left\{\mathcal{M}^{i} \cup i\right\}}\left[U^{j}(t)-z^{i}(t)\right]$. The inputs to the local LCF filter are $U^{j}(t)=H^{j \top} V^{j-1} y^{j}(t)$ and the output is the internal state $z^{i}(t)$. It is possible to show that by choosing a suitably small step size $\epsilon$ (as a function of the graph Laplacian), all internal states $z^{i}(t), i=1, \ldots, N$ converge to $y(t)=\frac{1}{N} \sum_{i=1}^{N} H^{i \top} V^{i-1} y^{i}(t)$. Due to the data fusion process, each MKF does not require a central computation sink nor an all-to-all topology of the estimation agents; the previously proposed decentralized Kalman filter architectures [4], [14] relied precisely on fully-connected sensor networks, increasing tremendously the cost of information exchange in the network. Moreover the DKF algorithm is naturally robust with respect to changes in the network topology, given that observability of the pair $\left(A, H^{c}\right)$ is preserved. Indeed it was shown in [11] that average consensus is a protocol which remains stable in the presence of switches in the graph topology, if connectivity is preserved.

\section{Diagnostic ARCHITECTURE AND BELIEF PROPAGATION}

This section is dedicated to the distributed fault detection and isolation architecture, based on the designed MKFs and on a distributed hypothesis testing algorithm, recently introduced with the name of belief consensus (see [10]).

\section{A. Fault detection}

Let us consider the innovation vector of a standard centralized KF (see, for instance, the information form KF in [4]) using all the measurements of the sensor network:

$$
i^{c}(t)=y^{c}(t)-H^{c} \bar{x}^{c}(t),
$$

where $y^{c}(t)=\operatorname{col}\left[y^{1}(t), \ldots, y^{M}(t)\right], \quad H^{c}=$ $\left[H^{1 \top} \ldots H^{M \top}\right]^{\top}$ and $\hat{x}_{c}(t)$ is the estimated state of the centralized KF. It is easy to show (see, for instance, [8]) that the mean value of (7) is affected by a modeling mismatch. Let us indeed introduce the centralized state 
estimation error $e^{c}(t) \triangleq x(t)-\hat{x}^{c}(t)$ and suppose that a fault described by a matrix $\Phi \in \mathcal{F}$ occurs. Then, the following expressions for $i^{c}(t)$ and $e^{c}(t)$ can be immediately derived: $i^{c}(t)=H^{c}\left[F e^{c}(t-1)+G \xi(t-1)\right]+H^{c} \Phi \hat{x}^{c}(t-1)+v^{c}(t)$, $e^{c}(t)=\left(I d-K H^{c}\right)\left[F e^{c}(t-1)+G \xi(t-1)\right]+(I d-$ $\left.K H^{c}\right) \Phi \hat{x}^{c}(t-1)$, where $K$ is the steady-state gain of the centralized $\mathrm{KF}, K=M H^{c^{\top}} V^{c-1} ; V^{c}$ is a block diagonal matrix (see Assumption 3) containing each sensor measurement variance. The expected values are:

$$
\begin{aligned}
E\left[i^{c}(t)\right]= & H^{c} F E\left[e^{c}(t-1)\right]+H^{c} \Phi \hat{x}^{c}(t-1) \\
E\left[e^{c}(t)\right]= & \left(I_{n}-K H^{c}\right) F E\left[e^{c}(t-1)\right] \\
& +\left(I_{n}-K H^{c}\right) \Phi \hat{x}^{c}(t-1) .
\end{aligned}
$$

The mean values of the innovation and estimation will drift from zero if a fault occurs. We can distribute the centralized KF on the sensor network following [12] and suppose that all the MKFs utilized by the estimation agents have reached a steady state (data fusion is still required for the measurement vector $y(t)$ ). If the $\mathrm{KF}$ is at steady state, then $K H^{c}=$ $M_{\mu} S$ (see also [12]); moreover, if all the estimators are endowed with common update equations, we have $\hat{x}^{c}(t)=$ $\hat{x}(t)$ at each node. Therefore $E\left[e^{c}(t)\right]=E[e(t)]$ at each node of the estimation network. On the other hand, notice that $E\left[i^{c}(t)\right]=\operatorname{col}\left[E\left[i^{1}(t), \ldots, E\left[i^{N}(t)\right]\right]\right.$, where $E\left[i^{i}(t)\right]=$ $H^{i}(F E[e(t-1)]+\Phi \hat{x}(t-1))$. The innovation mean value is thus naturally not equal at each node of the network: some form of synchronization of the estimation agents is therefore needed to reach a common decision. We propose the following:

\section{Fault detection procedure.}

1) Define thresholds $\eta^{i}(t)$, as functions of the local measurement noise variance and of term $H^{i} \Phi \hat{x}(t-1)$ in (8): such thresholds are needed to trigger a local alert.

2) Define an internal alarm signal $\nu^{i}(t)$ for each sensor. This signal is set to zero in nominal conditions; if $\| E\left[i^{i}(t)\|\geq\| \eta^{i}(t) \|\right.$, set $\nu^{i}(t)=1$.

3) Define for each sensor $\mathcal{E}^{i}, i=1, \ldots, N$, an internal state $q^{i}(t)$, representing the level of alarm throughout the network, $q^{i}(0)=0$, and $q(t)=$ $\operatorname{col}\left[q^{1}(t), \ldots, q^{N}(t)\right]$.

4) Synchronize the internal states using an LCF as outlined in Section III-B , using as inputs the internal alarm signals $\nu^{j}(t), j \in\left\{\mathcal{M}^{i} \cup i\right\}$.

5) Sensor $\mathcal{E}^{i}$ declares that a fault has occurred if $q^{i}(t)>$ $\alpha$, where $\alpha$ is a suitable threshold to be designed.

By the procedure above, a common detection decision is reached by averaging throughout the network the alarm level, avoiding as much as possible false alarms.

\section{B. Isolation of the fault}

Once the occurrence of a fault has been detected, each sensor can activate its own bank of isolation filters. ${ }^{1}$ From now on, we will denote with an index $j$ the quantities related to the isolation filter using model $F=A+\Phi^{j}, j=1, \ldots, N_{f}$. Consider again the innovation vector signal $i^{c j}(t)$ defined in (8), that would be generated by the $j$ th centralized KF using all the measurements of the network. As shown in [8], the covariance matrix of (8) is:

$$
\Lambda^{j}(t)=H^{c \top} P^{j}(t) H^{c}+V^{c},
$$

where $P^{j}(t)$ is the a priori state covariance matrix.

Definition 4.1: Define $h^{0}$ as the hypothesis that system (1) is in nominal condition; $P\left(h_{x}^{0} \mid Y_{t}^{c}\right)$ is the corresponding conditional probability, given the past measurement history $Y_{t}^{c} \triangleq \operatorname{col}\left[y^{c}(0), \ldots, y^{c}(t-1)\right]$. Moreover, we define $h^{j}$, for $j=1, \ldots, N_{x}$, as the hypothesis that system (1) is affected by the $j$ th fault $\Phi_{x}^{j}$; accordingly, $P\left(h_{x}^{j} \mid Y_{t}^{c}\right)$ is the conditional probability density function that the $j$ th fault has occurred at time-instant $t$, given the past measurement history $Y_{t}^{c}$.

The expression of the conditional density according to the the above definition is:

$$
p\left(y^{c} \mid Y_{t-1}^{c}, h_{x}^{j}\right)=C\left[\Lambda^{j}(t)\right] \exp \left[-\frac{1}{2} i^{c j \top}(t) \Lambda^{j}(t)^{-1} i^{c j}(t)\right]
$$

We can write:

$$
P\left(h_{x}^{j} \mid Y_{t}^{c}\right)=\frac{1}{\alpha} p\left(y^{c}(t) \mid h_{x}^{j}, Y_{t-1}^{c}\right) P\left(h_{x}^{j} \mid Y_{t-1}^{c}\right),
$$

where $\alpha=\sum_{k=1}^{N_{x}+1} P\left(h_{x}^{k} \mid Y_{t-1}^{c}\right)$ is a normalization factor. However, all innovations across the network are not available. By applying the matrix inversion lemma to (9), it is actually possible to distribute the computation of (10). In fact, consensus can be reached on the value of $i^{c j \top}(t) \Lambda^{j}(t)^{-1} i^{c j}(t)=i^{c j \top}(t)\left(V^{c-1}-\right.$ $\left.V^{-1} H\left(P(t)^{-1}+H^{\top} V^{c-1} H\right)^{-1} H^{\top} V^{c-1}\right) i^{c j}(t)$ by observing that $\left(P(t)^{-1}+H^{\top} V^{c-1} H\right)^{-1}=\left(P(t)^{-1}+S\right)^{-1}=$ $M_{\mu}(t)$ as in (6), and that $V^{i}$ is block diagonal. The computation of the determinant cannot be distributed, but it could be estimated by one centralized computation using the steady state values of $M_{\mu}$. It is though numerically infeasible to utilize the centralized probability of model $j$ being valid: the size of matrix (9) and of the innovation vector will result in possible ill-conditioning and too high values of the corresponding quadratic form. The only option is that of approximating (10) as a product of local densities:

$$
p\left(y^{c} \mid Y_{t-1}^{c}, h_{x}^{j}\right) \simeq \prod_{i=1}^{N} p\left(y^{i} \mid Y_{t-1}^{i}, h_{x}^{j}\right) .
$$

This means assuming that the innovations at each node are independent: such a hypothesis does not hold, due to the

\footnotetext{
${ }^{1}$ The problem of perfect simultaneous activation of the filters is not relevant: it is only necessary to produce locally another estimation, depending on the new activated filter gain and on the vector $y(t)$ which has been calculated at all times at all the sensors. Isolation will be performed by analyzing the innovations of each filter.
} 
average measurement utilized by the $j$-th MKF and to the presence of process noise. We can though substitute this strong assumption by supposing that, for any two estimation agents $\mathcal{E}^{i}, \mathcal{E}^{k}$, with $k \neq i$, the cross-correlations between the respective innovation vectors $i^{i}(t)$ and $i^{k}(t)$ have a sufficiently small magnitude, depending on the specific application under concern. This can be verified in the design stage by analyzing (9) at steady state. If this is possible, we can compute (12) at each node of the network, by performing abelief consensus algorithm [10]. In fact, defining

$$
Q(i) \triangleq \log \left[p\left(y^{i} \mid Y_{t-1}^{i}, h_{x}^{j}\right)\right]
$$

the network only has to reach consensus on $Q=$ $\frac{1}{N} \sum_{i=1}^{N} Q(i)$ and then find $p\left(y^{c} \mid Y_{t-1}^{c}, h_{x}^{j}\right)=\exp [N Q]$.

To sum up, if measurements are sufficiently uncorrelated in the sense specified above, then we are able to perform also a distributed diagnosis task based on the application of DKF and hypothesis testing. In Figure 2, a graphical description of the generic diagnosis agent is depicted.

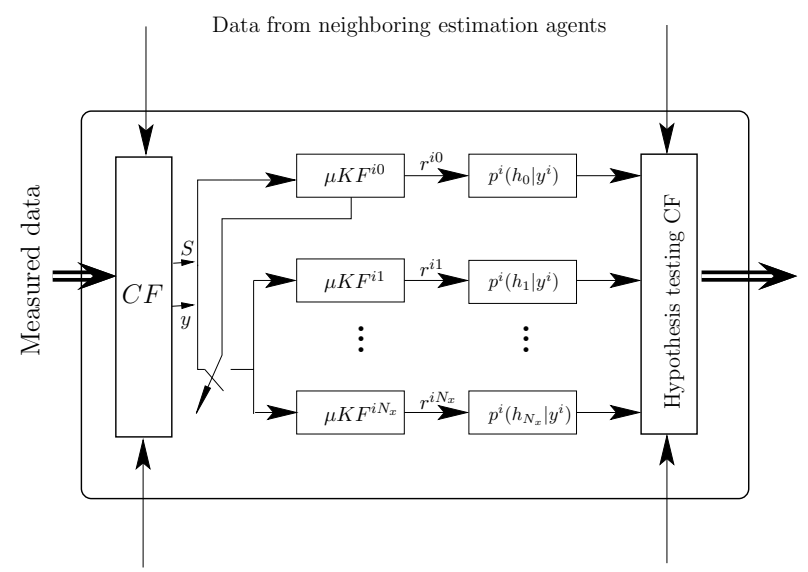

Data from neighboring estimation agents

Fig. 2. Diagnosis agent architecture.

\section{A SIMPLE APPLICATION EXAMPLE}

We consider a network of $N=20$ estimation agents in order to carry out FDI for system representing a moving object on a plane. The topology of the sensor network is depicted in Fig. 1. The nominal system is described by $x(t+$ 1) $=A x(t)+\xi(t)$, where

$$
A \triangleq\left[\begin{array}{cccccc}
1 & \delta & 0 & 0 & 0 & 0 \\
0 & 1-\frac{\delta \mu}{m} & \frac{\delta}{m} & 0 & 0 & 0 \\
0 & 0 & 1 & 0 & 0 & 0 \\
0 & 0 & 0 & 1 & \delta & 0 \\
0 & 0 & 0 & 0 & 1-\frac{\delta \mu}{m} & \frac{\delta}{m} \\
0 & 0 & 0 & 0 & 0 & 1
\end{array}\right]
$$

and $\delta=0.1 \mathrm{~s}$ is the sampling time; the mass of the vehicle $m$ is equal to $.75 \mathrm{Kg}$ and the friction coefficient $\mu=.15$. The process noise is $\xi \sim W G N(0, \Xi)$, where $X^{i}=$ $10^{-4} \operatorname{diag}(1,1,1,1)$; the initial a priori state covariance is $P=\operatorname{diag}(1, .1, .01,1, .1, .01)$. The state vector is initialized as $x(0)=\operatorname{col}[0,0, .1,0,0, .1]$. We assume that two types of faults $\left(N_{f}=2\right)$ can occur: $F^{1}$, given by the sign inversion of the force contributed by one of the actuators along the vertical direction, and $F^{2}$, causing the actuator governing the vehicles movement along the $y$ axis to get stuck. These faults can be described by (see (1)):

$\Phi^{1}=\left[\begin{array}{cccccc}0 & 0 & 0 & 0 & 0 & 0 \\ 0 & 0 & 0 & 0 & 0 & 0 \\ 0 & 0 & 0 & 0 & 0 & 0 \\ 0 & -\delta & 0 & 0 & 0 & 0 \\ 0 & -\frac{\delta}{m} & 0 & 0 & 0 & 0 \\ 0 & 0 & 0 & 0 & 0 & 0\end{array}\right], \Phi^{2}=\left[\begin{array}{cccccc}0 & 0 & 0 & 0 & 0 & 0 \\ 0 & 0 & 0 & 0 & 0 & 0 \\ 0 & 0 & 0 & 0 & 0 & 0 \\ 0 & 0 & 0 & 0 & 0 & 0 \\ 0 & 0 & 0 & 0 & \frac{\delta \mu}{m} & -\frac{\delta}{m} \\ 0 & 0 & 0 & 0 & 0 & 0\end{array}\right]$

Thus, every $\mathcal{E}^{i}, i=1, \ldots, N$ is provided with $N_{f}+1=3$ MKFs, each implementing the equations in (6) according to the aforementioned models of the fault. Such filters will be activated once a fault is detected using the nominal system model. We do not take into account the delays introduced by the physical distance between the nodes of the network. Each sensor is able to measure the position and velocity of the vehicle. The measurement noise is the same for all the sensors, and is $v^{i} \sim W G N\left(0, V^{i}\right)$, $V^{i}=10^{-1} \operatorname{diag}(1,10,1,10)$. Since the measurement noise covariance and the measurement matrices $H^{i}$ are constant over time, the network agreement on the average inverse covariance matrix $S$ is done off line. On the other hand, at each measurement sample the network calculates on-line the average measurement vector $y(t)$ by local implementation of a low-pass consensus filter. Running only one agreement step of such filter, in between MKF updates, is sufficient to guarantee tracking of the average measurement. The topology of the graph affects the consensus velocity, since it is related to the graph Laplacian eigenvalues. Two tests were considered; in the first, the model switches from the nominal one to the one corresponding to fault $F^{1}$ at $t=7 \mathrm{~s}$; in the second, the switch is to the model corresponding to fault $F^{2}$ at $t=7 \mathrm{~s}$. Checking that the resulting network measurements are sufficiently uncorrelated by finding the cross-correlation coefficients matrix, we can apply the proposed detection/isolation agorithm. The thresholds have been determined according to the noise covariance and the specific system: when the alarm internal state $q^{i}(t)$ is greater than 0.5, we declare the fault has occurred and start the isolation algorithm. At each step the measurement covariance is computed by every filter at node $i$, deriving the conditional probability that the $j$-th model is valid by running a second agreement step. We are testing the probability of hypothesis $h^{j}, j=0,1,2$ being true given the measurement $y(t)$. The results for the first simulations (occurrence of fault $F^{1}$ ) are shown in Figure 3, for the ensemble of nodes of the network. Figure 5 shows the results of the second case, where fault $F^{2}$ occurs; the reported results are for a series of 100 Monte Carlo simulations. In Figure 4 we report the behavior of the network detecting fault $F^{1}$ when agents $\mathcal{E}^{i}$, $i=3,5,9,15$ are implementing an unobservable model, being able to measure the velocity only with noise variance $V^{i}=10^{-2} \operatorname{diag}(.1, .1)$; this result refers to 10 Monte Carlo runs. It is interesting to note that despite the unobservability of a subset of implemented models, all the estimators are able to perform the test correctly. 

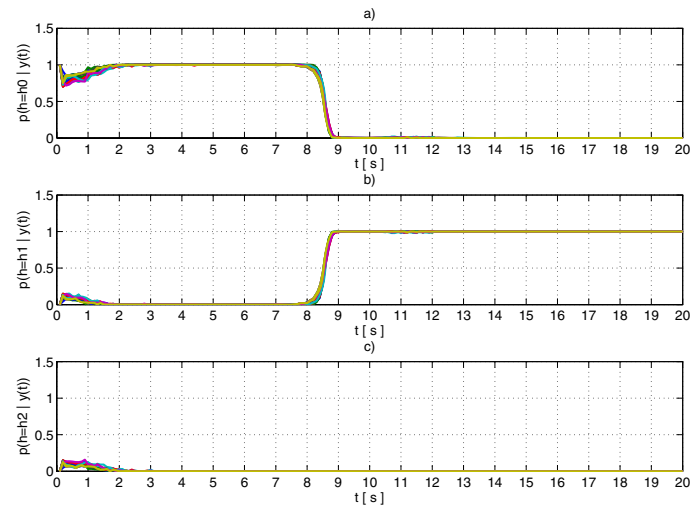

Fig. 3. Simulation 1: (a) conditional probability of the nominal model being valid; (b) conditional probability of the faulty model $F^{1}$ being valid; (c) conditional probability of the faulty model $F^{2}$ being valid.
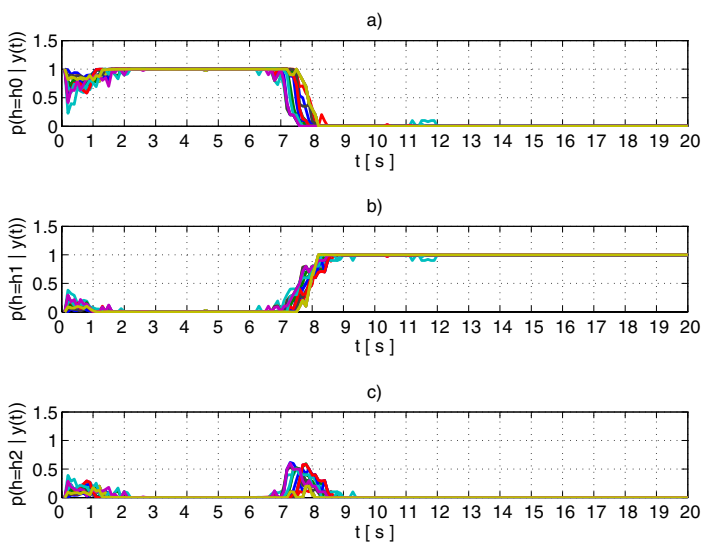

Fig. 4. Simulation 1: (a) conditional probability of the nominal model being valid; (b) conditional probability of the faulty model $F^{1}$ being valid; (c) conditional probability of the faulty model $F^{2}$ being valid.
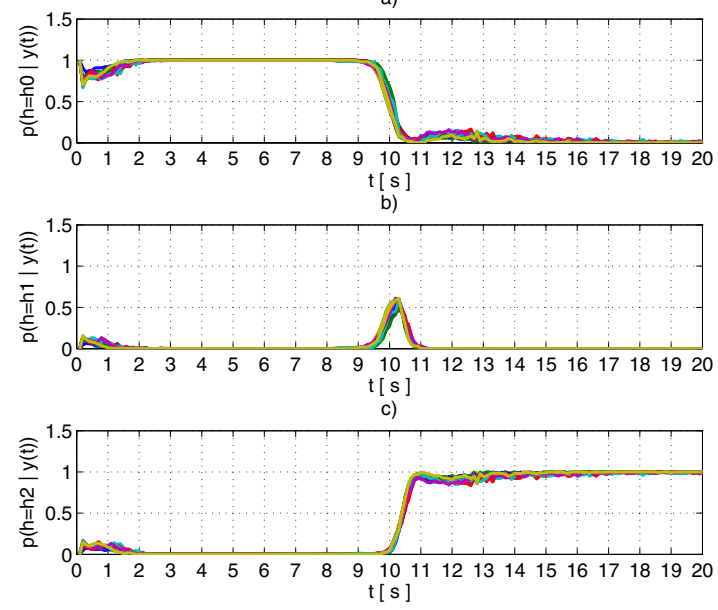

Fig. 5. Simulation 2: (a) conditional probability of the nominal model being valid; (b) conditional probability of the faulty model $F^{1}$ being valid; (c) conditional probability of the faulty model $F^{2}$ being valid.

\section{Vi. Conclusions}

In this paper, the problem of distributed fault diagnosis for dynamic systems using sensor networks has been considered. For the first time, consensus algorithms have been used towards the agreeement of the estimates provided by the distributed estimation agents and to devise a distributed hypothesis testing method that relies on a belief consensus technique. An algorithm for fault detection and isolation has been proposed and early simulation results have been reported showing the potentialities of the proposed methodology.

\section{REFERENCES}

[1] E. Benazera and S. Narasimhan, "An extension to the kalman filter for an improved detection of unknown behavior," in Proc. of the IEEE American Control Conference, Portland, June 2005, pp. 1039-1041.

[2] B. Bollobás, Modern Graph Theory, ser. Graduate Texts in Mathematics. Springer-Verlag, 1998, vol. 184.

[3] J. Chen and R. J. Patton, Robust Model-Based Fault Diagnosis for Dynamic Systems. Kluwer Academic Publishers, London, 1999.

[4] W. H. Chung and J. L. Speyer, "A general framework for decentralized estimation," in Proceedings of the American Control Conference, Washington, Whashington, 1996, pp. 2931-2935.

[5] W. H. Chung, J. L. Speyer, and R. H. Chen, "A decentralized fault detection filter," ASME J. of Dynamic Systems, Measurement and Control, vol. 123, no. 2, pp. 237-247, 2001.

[6] P. M. Frank, "Fault diagnosis in dynamic systems using analytical and knowledge-based redundancy - A survey and some new results," Automatica, vol. 26, pp. 459-474, 1990.

[7] J. J. Gertler, Fault Detection and Diagnosis in Engineering Systems. Marcel Dekker, New York, 1998.

[8] P. S. Maybeck and P. D. Hanlon, "Performance enhancement of a multiple model adaptive estimator," IEEE Trans. on Aerospace and Electronic Systems, vol. 31, no. 4, pp. 1240-1254, 1995.

[9] R. Olfati-Saber, "Distributed Kalman filter with embedded consensus filters," in Proc. of the 44th IEEE Conference on Decision and Control, 2005 and 2005 European Control Conference, Sevilla, 2005, pp. 81798184.

[10] R. Olfati-Saber, E. Franco, E. Frazzoli, and J. S. Shamma, "Belief consensus and distributed hypothesis testing in sensor networks," in Network Embedded Sensing and Control, LNCIS 331, P. Antsaklis and P. Tabuada, Eds. Univ. of Notre Dame, IN: Springer-Verlag, October 2005, pp. 169-182.

[11] R. Olfati-Saber and R. M. Murray, "Consensus problems in networks of agents with switching topology and time-delays," IEEE Trans. on Automatic Control, vol. 49, no. 9, pp. 1520-1533, 2004.

[12] R. Olfati-Saber and J. S. Shamma, "Consensus filters for sensor networks and distributed sensor fusion," in Proc. of the 44th IEEE Conference on Decision and Control, 2005 and 2005 European Control Conference, Sevilla, 2005, pp. 6698-6703.

[13] J. Pearl, Probabilistic reasoning in intelligent systems: networks of plausible inference. Morgan Kaufmann, 1988.

[14] B. S. Rao, H. F. Durrant-Whyte, and J. A. Sheen, "A fully decentralized multi-sensor system for tracking and surveillance," Int. Journal of Robotics Research, vol. 12, no. 1, pp. 20-44, 1993.

[15] S. Roumeliotis, G. S. Sukhatme, and G. A. Bekey, "Fault detection and identification in a mobile robot using multiple model estimation," in Proc. of the IEEE International Conference on Robotics an Automation, Leuven, May 1998, pp. 2223-2228.

[16] A. T. Vemuri and M. M. Polycarpou, "On-line approximation based methods for robust fault detection," in 13-th IFAC World Congress, San Francisco, CA, 1996.

[17] X. Zhang, M. Polycarpou, and T. Parisini, "A robust detection and isolation scheme for abrupt and incipient faults in nonlinear systems," IEEE Trans. on Automatic Control, vol. 47, pp. 576-593, 2002. 